\title{
Impedance Behaviors for Two-handed Manipulation: Design and Experiments
}

\author{
Thomas Wimböck, Christian Ott, and Gerd Hirzinger \\ Institute of Robotics and Mechatronics \\ German Aerospace Center (DLR) \\ Oberpfaffenhofen, 82234 Wessling, Germany \\ \{thomas.wimboeck, christian.ott\}edlr.de
}

\begin{abstract}
The control of humanoid manipulators is very challenging due to the large number of degrees of freedom and the resulting redundancy. Using joint-level control complex planning algorithms are needed to accomplish tasks. For intuitive operation and hence short development times of applications high-level control interfaces are needed. Furthermore, for many tasks it is desirable to define an impedance behavior in task space. In this paper a flexible control law is proposed which offers object-level impedances for two-handed manipulation. The controller structure is based on the wellknown compliance control law. The main contributions of this work are the way how to combine several potential functions for two-handed manipulation and the experimental validation of hand-arm coordination. The controller is implemented on DLR's humanoid manipulator Justin and its performance is demonstrated experimentally by unscrewing a can and motion of a grasped box.
\end{abstract}

\section{INTRODUCTION}

Various kinds of anthropomorphic robot arms and hands have been developed in the past. Since most systems were designed as separate devices, the availability of combined hand-arm systems is still very low at the moment. Consequently, most control algorithms were hence developed for hands or arms separately.

Joint velocity, torque range, inertia, kinematics, workspace, and its end-effector distinguish robot arms from hands. Arms are characterized by serial chains that have low maximum joint velocity, high joint torques, a large workspace, and high inertia. The fingers of a hand represent several cooperating robots with each typically less than 6 degrees of freedom (DOF). The inertia of robot fingers is very low and the workspace is limited. The problems related with separate control designs for hands and arms are pointed out by Bae et al. [1]. A control law is proposed for manipulation in 2D. Orientation, position control, and grasping control are superimposed. Nagai and Yoshikawa [2] specify impedances for the tool center point (TCP) of the arm and the grasped object including an internal force controller to ensure grasp stability. The control law was validated experimentally on a simple setup with $1 \mathrm{D}$ object motion. A hand-arm control law including the partitioning of the contribution of the hand and the arm towards task completion is shown by Melchiorri and Salisbury [3]. The theory was supported by simulations and an experiment with one finger attached to an arm.
Two-handed manipulation involving dextrous hands is particularly of interest for humanoid robots. Basic autonomous manipulation skills as well as teleoperated manipulation have been presented, e.g., with NASA's Robonaut [4] and the Japanese humanoid robot HRP [5]. Platt Jr. et al. [6] describes a control basis that is suitable for force-based interactions. Therein, policies that maintain wrench closure constraints during manipulation are combined with reinforcement learning and experiments are performed with Dexter. On the platform WENDY a behavior was implemented that allows the user to interact with the robot during task execution using its force-/torque sensors [7].

Intuitive and fast operation of a high DOF system demands a high-level interface. E. g. commanding only the object motion of a grasped object facilitates the development of applications. Furthermore, many tasks are very difficult to accomplish with one arm, like opening of a bottle. A second hand is needed to fix the bottle while unscrewing its lid. Figure 1 shows DLR's humanoid manipulator Justin [8] before opening a can.

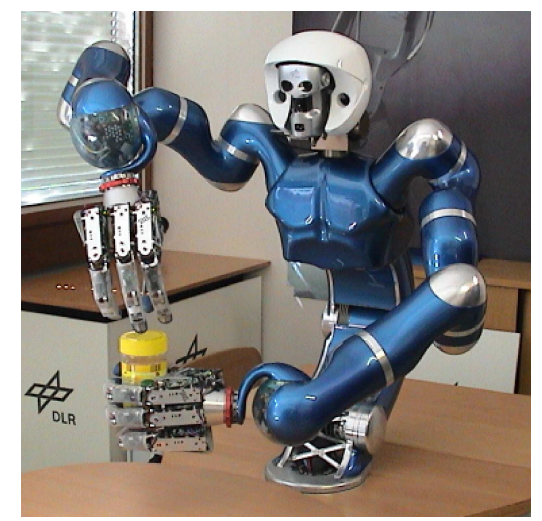

Fig. 1. DLR's humanoid manipulator Justin before unscrewing a can.

Equipped with two arms and two dextrous hands a wide range of tasks can be accomplished. There is no unique solution how a task can be achieved. A can may be opened with power grasps or fingertip grasps for both hands or a combination of both grasp types. In this paper we want to present a controller structure that implements a changeable behavior enabling the robot to fulfill a large set of possible tasks. In contrast to control approaches designed for general 
multi-robot-systems, the proposed impedance behaviors are custom-built for humanoid robots with two arms and two hands.

Section II treats the design of the Cartesian impedance behavior. In Section III two experiments validate the performance of the proposed control laws.

\section{CARTESiAn IMPEDANCE BehaVIORS}

In many robotic applications using a single manipulator the desired Cartesian impedance behavior is typically chosen as some sort of mass-spring-damper-like system. Thereby, inertia shaping is a difficult issue. It is well known that for shaping of the apparent inertia generally a measurement of the external force is needed. As a result forces which act at a point of the robot where they cannot be measured will experience a different impedance behavior than desired. Instead, it is often sufficient in practice to restrict the desired behavior to an impedance in which only the stiffness and the damping must be shaped while one can keep the natural inertia unchanged, resulting in a compliance control problem. Within this paper we also aim at impedance behaviors without inertia shaping. Such a Cartesian impedance behavior for a single arm can be seen as a viscoelastic spatial spring attached to the end-effector. Many works in the robotics community already treated the design of such spatial springs. Caccavale and Natale investigated the rotational part of the stiffness based on different orientation representations [9], [10]. Spatial stiffness controllers including a coupling stiffness term between translation and rotation were proposed in the works of Fasse and Zhang [11]-[13]. Stramigioli analyzed the power flow for these springs when the rest length ${ }^{1}$ and the stiffness values are subject to changes [14]. One possible way how to utilize the spatial stiffness for the control of multi-fingered hands was presented in form of the so-called Intrinsically Passive Controller (IPC) in [15].

An important feature for all these stiffness implementations is the fact that there exists a potential function $V_{s}$ associated with the spatial spring. It is well known that the existence of a lower bounded potential function is closely related to the stability and passivity properties of the closed loop system.

In the following such kind of viscoelastic spatial springs will be used as building blocks for the definition of useful Cartesian impedance behaviors for multi-fingered twohanded (i.e. humanoid) manipulator systems. The potential function according to a spatial spring connected between the frames $^{2} \boldsymbol{H}_{1} \in S E(3)$ and $\boldsymbol{H}_{2} \in S E(3)$ will be denoted by $V_{s}\left(\boldsymbol{H}_{1}, \boldsymbol{H}_{2}, \mathcal{K}\right)$, where the symbol $\mathcal{K}$ represents the relevant parameters according to the chosen implementation of the spatial spring (e.g. the rest length and the values of the stiffness matrix). Appropriate choices for the potential

\footnotetext{
${ }^{1}$ For the ease of discussion the relative pose between the two connection frames of the spring in the relaxed configuration will be simply called rest length within this paper.

${ }^{2}$ Here, $S E(3)$ denotes the special Euclidean group and an element $\boldsymbol{H} \in$ $S E(3)$ can be written as $\boldsymbol{H}=[\boldsymbol{R}, \boldsymbol{p}] \in \mathbb{R}^{3 \times 4}$, with a rotation matrix $\boldsymbol{R} \in S O(3)$ and a translation vector $\boldsymbol{p} \in \mathbb{R}^{3}$.
}

function $V_{s}$ can be easily found in the references cited above, and therefore will not be repeated herein in detail. An example implementation is shown in the appendix.

After discussing the implementation of the impedance controller in Section II-A, some useful impedance behaviors for a system consisting of two arms are discussed in Section II-B. Then, in Section II-C an object-level impedance controller for an hand-arm-system is discussed. Furthermore, a combination of these impedances for two-handed object manipulation is presented in Section II-D, which is particularly useful for humanoid robots. Finally, in Section II-E the redundancy treatment is discussed.

\section{A. Implementation of the Impedance Behaviors}

The considered dynamical model for a manipulator with $n$ degrees of freedom has the form of a rigid multi-bodysystem and is written as

$$
\boldsymbol{M}(\boldsymbol{\theta}) \ddot{\boldsymbol{\theta}}+\boldsymbol{C}(\boldsymbol{\theta}, \dot{\boldsymbol{\theta}}) \dot{\boldsymbol{\theta}}+\boldsymbol{g}(\boldsymbol{\theta})=\boldsymbol{\tau}+\boldsymbol{\tau}_{\text {ext }},
$$

where $\theta \in \mathbb{R}^{n}$ is the vector of joint coordinates from all the subsystems of the robot (arms, hands, torso, etc ...). The matrix $\boldsymbol{M}(\boldsymbol{\theta}) \in \mathbb{R}^{n \times n}$ is the symmetric and positive definite inertia matrix, $\boldsymbol{C}(\boldsymbol{\theta}, \dot{\boldsymbol{\theta}}) \dot{\boldsymbol{\theta}} \in \mathbb{R}^{n}$ contains the centrifugal and Coriolis components, and $\boldsymbol{g}(\boldsymbol{\theta}) \in \mathbb{R}^{n}$ is the vector of generalized $^{3}$ gravity forces. The vector $\tau \in \mathbb{R}^{n}$ contains the generalized actuator forces which are considered as the control inputs. Finally, $\boldsymbol{\tau}_{\text {ext }} \in \mathbb{R}^{n}$ contains external generalized forces acting on the robot. In order to focus the presentation on the multi-body part of the dynamics, other physical effects like link and joint flexibility as well as joint friction are neglected. Clearly, in practice it is important to consider friction and thus to augment the controller by an appropriate friction compensation.

For the implementation of all the presented impedance behaviors then the well-known compliance control law

$$
\boldsymbol{\tau}=\boldsymbol{g}(\boldsymbol{\theta})-\left(\frac{\partial V(\boldsymbol{\theta})}{\partial \boldsymbol{\theta}}\right)^{T}-\boldsymbol{D}(\boldsymbol{\theta}) \dot{\boldsymbol{\theta}}
$$

is assumed. Herein, $\boldsymbol{D}(\boldsymbol{\theta})$ is a positive semi-definite damping matrix. The overall potential function $V(\boldsymbol{\theta})$ is chosen according to the impedance behaviors described in the following sections. It basically consists of the sum of the individual potential functions of all the stiffness components. While the control law (2) represents a classical controller structure in robotics, the way how to combine different (viscoelastic) spatial springs (i.e. how to setup the potential function) in an appropriate way for two-handed humanoid manipulation is the main contribution of the present paper.

From a passivity point of view, the damping matrix $\boldsymbol{D}(\boldsymbol{\theta})$ can be any positive semi-definite matrix. Herein it is chosen such that the Cartesian closed loop behavior is critically damped. This can be achieved via a double diagonalization ${ }^{4}$

\footnotetext{
${ }^{3}$ Depending on the type of joint (rotational or prismatic) this generalized force is either a torque or a force.

${ }^{4}$ Given a symmetric and positive definite matrix $\boldsymbol{A} \in \mathbb{R}^{n \times n}$ and a symmetric matrix $\boldsymbol{B} \in \mathbb{R}^{n \times n}$. Then one can find a non-singular matrix $\boldsymbol{Q} \in \mathbb{R}^{n \times n}$ and a diagonal matrix $\boldsymbol{B}_{0} \in \mathbb{R}^{n \times n}$, such that $\boldsymbol{Q}^{T} \boldsymbol{Q}=\boldsymbol{A}$ and $\boldsymbol{B}=\boldsymbol{Q}^{T} \boldsymbol{B}_{0} \boldsymbol{Q}[16]$.
} 
of the inertia matrix $M(\theta)$ and the Hessian of the potential function $\frac{\partial^{2} V(\boldsymbol{\theta})}{\partial \boldsymbol{\theta}^{2}}$.

\section{B. Coordinated Two-Armed Manipulation}

For a two-arm system an impedance behavior could simply be designed by using two spatial springs $\mathcal{K}_{r}$ and $\mathcal{K}_{l}$ for the right and the left arm, which connect the end-effectors to the virtual equilibrium frames $\boldsymbol{H}_{r, d}$ and $\boldsymbol{H}_{l, d}$, respectively. If the springs are implemented as complete $6 \mathrm{D}$-springs (i.e. with full rank $(6 \times 6)$-stiffness matrices), then the complete Cartesian motion of the arms can be influenced already via only these two springs. For some applications it is useful if some part of the motion is instead defined via an additional coupling spring $\mathcal{K}_{c}$ between the arms. Clearly, in such a configuration (as shown in Fig. 2) both the rest lengths as well as the stiffness values of the individual springs and the coupling spring should be chosen in a compatible way such that the springs do not interfere with each other. The complete potential function for such a two-arm impedance is given by

$$
\begin{aligned}
V(\boldsymbol{\theta})= & V_{s}\left(\boldsymbol{H}_{r}(\boldsymbol{\theta}), \boldsymbol{H}_{r, d}, \mathcal{K}_{r}\right)+V_{s}\left(\boldsymbol{H}_{l}(\boldsymbol{\theta}), \boldsymbol{H}_{l, d}, \mathcal{K}_{l}\right)+ \\
& V_{s}\left(\boldsymbol{H}_{r}(\boldsymbol{\theta}), \boldsymbol{H}_{l}(\boldsymbol{\theta}), \mathcal{K}_{c}\right) .
\end{aligned}
$$

With an impedance structure as depicted in Fig. 2 one can thus implement different behaviors, ranging from an independent control of the arms via $\mathcal{K}_{r}$ and $\mathcal{K}_{l}$ to a pure coupling based on $\mathcal{K}_{c}$. One disadvantage of this structure, however, is the fact that the springs should not be designed independently, but in a compatible way as explained above.

Instead of the two individual springs attached to the end-effectors one can define a virtual object frame $\boldsymbol{H}_{o}\left(\boldsymbol{H}_{r}(\boldsymbol{\theta}), \boldsymbol{H}_{l}(\boldsymbol{\theta})\right)$ depending on the two end-effector frames as sketched in Fig. 3. Such a virtual object was used by Natale in [10]. The object frame was chosen as $\boldsymbol{H}_{o}=\left[\boldsymbol{R}_{o}, \boldsymbol{p}_{o}\right]$ with $\boldsymbol{p}_{o}=\frac{1}{2}\left(\boldsymbol{p}_{r}+\boldsymbol{p}_{l}\right)$ and $\boldsymbol{R}_{o}=\boldsymbol{R}_{r} \overline{\boldsymbol{R}}_{r l}$, where $\overline{\boldsymbol{R}}_{r l}$ denotes $a$ half ${ }^{5}$ of the rotation matrix $\boldsymbol{R}_{r}^{-1} \boldsymbol{R}_{l}$. This virtual object is connected via a spatial spring $\mathcal{K}_{o}$ to a virtual equilibrium pose $\boldsymbol{H}_{o, d}$. In combination with the coupling stiffness, one can intuitively define an impedance behavior which is useful for grasping large objects with two arms. The relevant potential function is given by

$$
\begin{aligned}
V(\boldsymbol{\theta})= & V_{s}\left(\boldsymbol{H}_{o}\left(\boldsymbol{H}_{r}(\boldsymbol{\theta}), \boldsymbol{H}_{l}(\boldsymbol{\theta})\right), \boldsymbol{H}_{o, d}, \mathcal{K}_{o}\right)+ \\
& V_{s}\left(\boldsymbol{H}_{r}(\boldsymbol{\theta}), \boldsymbol{H}_{l}(\boldsymbol{\theta}), \mathcal{K}_{c}\right) .
\end{aligned}
$$

\section{Object-Level Control of a single Hand-Arm-System}

In [17] we presented a passivity-based object-level controller for a multifingered hand similar to [15] which is based on the compliance control law (2). This controller can be applied also to combined hand-arm-systems, e. g. dextrous hands mounted on an arm. This controller will be combined with the impedances from Section II-B in the following to build complete two-hand impedance behaviors. Therefore, the controller from [17] is reviewed shortly. Notice that

\footnotetext{
${ }^{5}$ This halving of the rotation matrix can easily be done using an angle-axis representation.
}

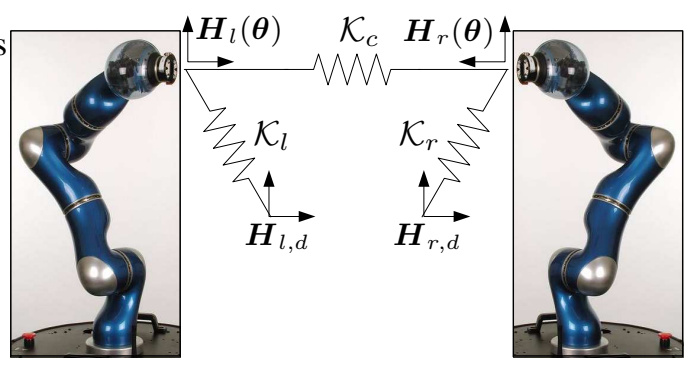

Fig. 2. Two-arm impedance behavior by adding a coupling spring to the individual arm springs.

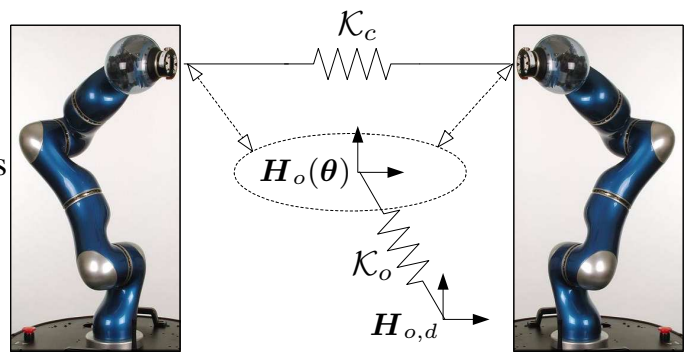

Fig. 3. Two-arm impedance behavior by combining a coupling spring with an object level spring.

herein $\boldsymbol{\theta}$ contains both the joint angles of the hand and of the arm.

It is assumed that the $N$ fingertips of each hand are in contact with the object. For point contacts with friction (PCWF), the held object could be manipulated using only hand joints in 3D-space if $N \geq 3$ and if each finger has a configuration space of dimension $\geq 3$. However the workspace of the hand is limited. The typically large range of the arm increases the workspace heavily. Instead of controlling each TCP of the fingers, object-level control is employed. It has in general the advantages that it is easy to define grasp forces, to specify forces acting on the object, and to avoid unnecessarily high internal forces [18]. The dimension of the task space is $6+M$, with $M$ the dimensions related to internal forces. The redundancy of a hand-arm-system w.r.t to the task is very high. The treatment of redundancy will be described later in Section II-D.

We assume furthermore that

- The internal forces provided by the controller are chosen to be sufficient such that the friction constraints are fulfilled for all contact points (no sliding).

- In order to allow 6D object motion the contact between the object and the hand are restricted to the fingertips (no palm contact).

- The object geometry is convex.

In contrast to [15] the object frame is defined uniquely by the positions of the fingertips. We propose a frame which is attached to the center of the fingertip positions

$$
\boldsymbol{x}_{h o}(\boldsymbol{\theta})=\frac{1}{N} \sum_{i=1}^{N} \boldsymbol{x}_{i}(\boldsymbol{\theta}),
$$




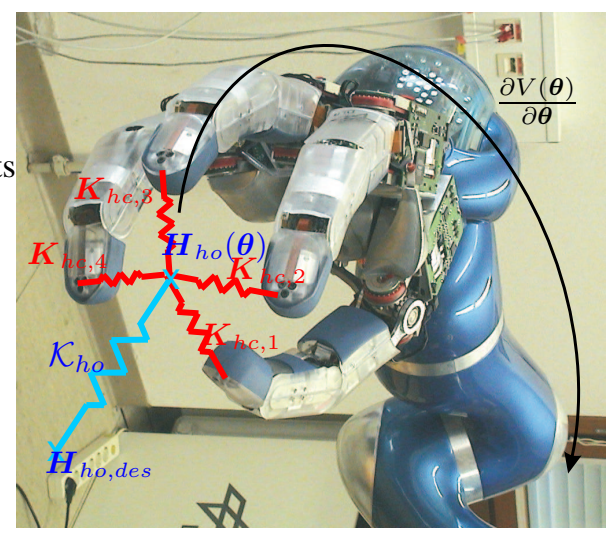

Fig. 4. DLR Hand II superimposed by the virtual springs defined by the potential functions in equation (10).

where $\boldsymbol{x}_{i}(\boldsymbol{\theta}) \in \mathbb{R}^{3}$ represents the Cartesian fingertip position for finger $i$ w.r.t the base of the arm. For simplicity we omit the dependence on $\boldsymbol{\theta}$ in this section. The orientation $\boldsymbol{R}_{h o}=\left[\boldsymbol{r}_{1, h o}, \boldsymbol{r}_{2, h o}, \boldsymbol{r}_{3, h o}\right]$ is defined based on the Cartesian fingertip positions as well. For $N=4$ fingers the unit vector $\boldsymbol{r}_{1, h o}$ is defined in the plane spanned by the vectors being defined by the connections between fingers 1 and 3 and between fingers 2 and 4 , that is

$$
\tilde{\boldsymbol{r}}_{1, h o}=\frac{\boldsymbol{x}_{1}-\boldsymbol{x}_{3}}{\left\|\boldsymbol{x}_{1}-\boldsymbol{x}_{3}\right\|}+\frac{\boldsymbol{x}_{2}-\boldsymbol{x}_{4}}{\left\|\boldsymbol{x}_{2}-\boldsymbol{x}_{4}\right\|}, \quad \boldsymbol{r}_{1, h o}=\frac{\tilde{\boldsymbol{r}}_{1, h o}}{\left\|\tilde{\boldsymbol{r}}_{1, h o}\right\|} .
$$

The unit vector $\boldsymbol{r}_{3, h o}$ is defined perpendicular to this plane and $\boldsymbol{r}_{2, h o}$ is defined such that $\boldsymbol{r}_{h o}$ belongs to $\mathrm{SO}(3)$ :

$$
\begin{gathered}
\tilde{\boldsymbol{r}}_{3, h o}=\widehat{\boldsymbol{x}_{1}-\boldsymbol{x}_{3}}\left(\boldsymbol{x}_{2}-\boldsymbol{x}_{4}\right), \quad \boldsymbol{r}_{3, h o}=\frac{\tilde{\boldsymbol{r}}_{3, h o}}{\left\|\tilde{\boldsymbol{r}}_{3, h o}\right\|}, \\
\boldsymbol{r}_{2, h o}=\widehat{\boldsymbol{r}}_{3, h o} \boldsymbol{r}_{1, h o},
\end{gathered}
$$

with the skew-symmetric operator

$$
\widehat{\boldsymbol{w}}: \mathbb{R}^{3} \rightarrow \mathbb{R}^{3 \times 3}, \quad \widehat{\boldsymbol{w}}=\left[\begin{array}{rrr}
0, & -w_{3}, & w_{2} \\
w_{3}, & 0, & -w_{1} \\
-w_{2}, & w_{1}, & 0
\end{array}\right] .
$$

The virtual object frame $\boldsymbol{H}_{h o}$ can be stacked to $\boldsymbol{H}_{h o}=$ $\left[\boldsymbol{R}_{h o}, \boldsymbol{x}_{h o}\right] \in \mathrm{SE}(3)$. Note that this representation has singularities if $\boldsymbol{x}_{j}-\boldsymbol{x}_{j+2}=0$ for $j=1 \vee j=2$ or if $\left(\boldsymbol{x}_{1}-\boldsymbol{x}_{3}\right) \|\left(\boldsymbol{x}_{2}-\boldsymbol{x}_{4}\right)$. For common convex objects like boxes, cylinders or spheres these singularities pose no problem. If we want to extend to more than four fingers we can make sure that all elements of $\boldsymbol{H}_{h o}$ are a function of all fingertip positions. The virtual object frame is related to the real object frame if we assume that the relative contact points between the fingertips and the object do not change (neglecting rolling effects).

In addition to an impedance related to the object pose, impedances to realize grasping forces are added. These impedances connect each fingertip position $\boldsymbol{x}_{i}$ with the virtual object frame (compare Fig. 4). The potential is chosen to be spherical for each fingertip:

$$
V_{h c}\left(\boldsymbol{\theta}, \mathcal{K}_{h c}\right)=\frac{1}{2} \sum_{i=1}^{N} K_{h c, i}\left[\left\|\Delta \boldsymbol{x}_{i}\right\|-l_{i, d}\right]^{2},
$$

with $\Delta \boldsymbol{x}_{i}=\boldsymbol{x}_{i}-\boldsymbol{x}_{h o}$ as the distance from the fingertip $i$ to the virtual object frame $\boldsymbol{x}_{h o}$, and $K_{h c, i}>0$ the corresponding connecting stiffness. The value $l_{i, d}$ corresponds to the rest length of the connecting stiffness. We summarize $K_{h c, i}$ and $l_{d, i}$ for $i=1, \ldots, N$ in $\mathcal{K}_{h c}$.

The desired potential for the object-level control law is composed of the potentials to derive the spatial object stiffness and the connecting stiffness, and thus is given by

$$
V(\boldsymbol{\theta})=V_{s}\left(\boldsymbol{H}_{h o}(\boldsymbol{\theta}), \boldsymbol{H}_{h o, d}, \mathcal{K}_{h o}\right)+V_{h c}\left(\boldsymbol{\theta}, \mathcal{K}_{h c}\right) .
$$

Inserting this potential into equation (2) a control law is obtained which generates the control torques for the arm and the hand. However, since the grasping forces sum up to zero at the end-effector of the arm, the internal forces are not mapped onto the arm.

\section{Coordinated Two-Handed Manipulation}

The impedances from the previous two subsections serve as a basis to generate impedance behaviors for two-handed manipulation. Based on the finger configuration of the hands two virtual object frames $\boldsymbol{H}_{o, r}(\boldsymbol{\theta})$ and $\boldsymbol{H}_{o, l}(\boldsymbol{\theta})$ are defined for the right and the left hand, respectively. The two-arm object impedances from Section II-B are then attached to these virtual objects, while in Section II-B they were attached to the end-effectors of the two arms. In combination with the connecting springs for realizing the grasp force from Section II-C intuitive impedance behaviors for the complete two-armhand-system are obtained. In Fig. 5 it is outlined how the object-level potential (4) (cf. Fig. 3) is extended.

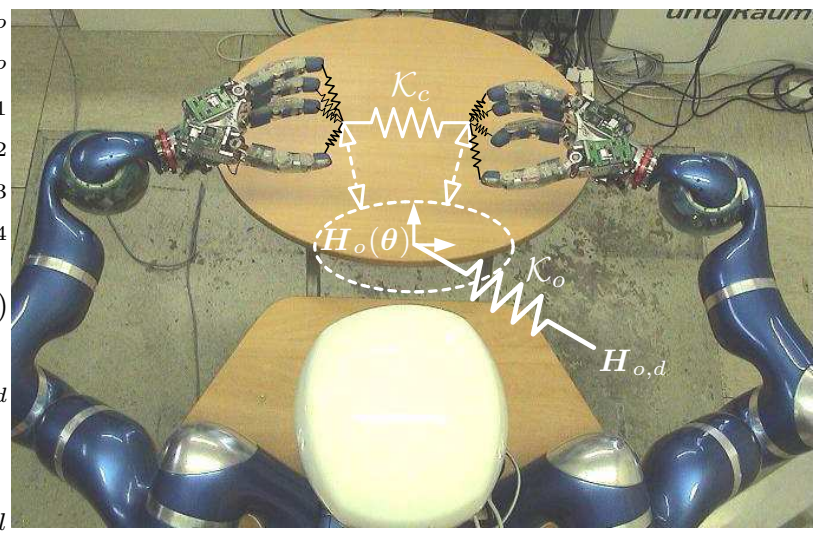

Fig. 5. Two-hand impedance behavior by combining the object-level impedances of the hands and the arms.

The potential functions for the impedances which generalize (3) and (4) to two-handed manipulation are given by

$$
\begin{aligned}
V_{I}(\boldsymbol{\theta})= & V_{s}\left(\boldsymbol{H}_{o, r}(\boldsymbol{\theta}), \boldsymbol{H}_{(o, r), d}, \mathcal{K}_{r}\right)+ \\
& V_{s}\left(\boldsymbol{H}_{o, l}(\boldsymbol{\theta}), \boldsymbol{H}_{(o, l), d}, \mathcal{K}_{l}\right)+ \\
& V_{s}\left(\boldsymbol{H}_{o, r}(\boldsymbol{\theta}), \boldsymbol{H}_{o, l}(\boldsymbol{\theta}), \mathcal{K}_{c}\right) \\
& V_{h c, r}\left(\boldsymbol{\theta}, \mathcal{K}_{h c, r}\right)+V_{h c, l}\left(\boldsymbol{\theta}, \mathcal{K}_{h c, l}\right),
\end{aligned}
$$


and

$$
\begin{aligned}
V_{O}(\boldsymbol{\theta})= & V_{s}\left(\boldsymbol{H}_{o}\left(\boldsymbol{H}_{o, r}(\boldsymbol{\theta}), \boldsymbol{H}_{o, l}(\boldsymbol{\theta})\right), \boldsymbol{H}_{o, d}, \mathcal{K}_{o}\right)+ \\
& V_{s}\left(\boldsymbol{H}_{o, r}(\boldsymbol{\theta}), \boldsymbol{H}_{o, l}(\boldsymbol{\theta}), \mathcal{K}_{c}\right)+ \\
& V_{h c, r}\left(\boldsymbol{\theta}, \mathcal{K}_{h c, r}\right)+V_{h c, l}\left(\boldsymbol{\theta}, \mathcal{K}_{h c, l}\right)
\end{aligned}
$$

where $V_{h c, r}\left(\boldsymbol{\theta}, \mathcal{K}_{h c, r}\right)$ and $V_{h c, l}\left(\boldsymbol{\theta}, \mathcal{K}_{h c, l}\right)$ are the potential functions of the finger connection springs according to (9) for the right and the left hand, respectively. Using these potential functions together with (2) coordinated control laws for a hand-arm-system are achieved.

\section{E. Redundancy Treatment}

Up to now only the Cartesian impedance behavior of the robot was considered. Clearly, in addition to the Cartesian behavior also the control of the nullspace behavior is important for kinematically redundant hand-arm-systems. Therefore, many different objectives like, e.g., the optimization of a manipulability index or an additional obstacle avoidance potential are possible. Herein, we only consider the avoidance of joint limits. Notice that this is particularly important for a combined hand-arm-system, since the workspace of the arm and the hand are quite different.

One simple, but effective, possibility to implement an impedance behavior for joint limit avoidance is to use another potential function $V_{l}(\boldsymbol{\theta})$ in superposition with (10),(12) or (11). Let $\theta_{i}$ denote the $i^{\text {th }}$ element of $\boldsymbol{\theta}$ and $\theta_{i, \min }$ and $\theta_{i, \max }$ its minimum and maximum value. Let further denote $\delta_{i} \in \mathbb{R}^{+}$and $k_{i} \in \mathbb{R}^{+}$a safety distance and a joint-limit stiffness value for the $i^{\text {th }}$ coordinate, then one can use a repelling potential function of the form

$$
\begin{aligned}
V_{l}(\boldsymbol{\theta}) & =\frac{1}{2} \sum_{i=1}^{n} k_{i} e_{i}\left(\theta_{i}\right)^{2} \\
e_{i}\left(\theta_{i}\right) & = \begin{cases}\theta_{i}-\left(\theta_{i, \text { max }}-\delta_{i}\right) & \text { for } \theta_{i}>\left(\theta_{i, \max }-\delta_{i}\right), \\
\theta_{i}-\left(\theta_{i, \text { min }}+\delta_{i}\right) & \text { for } \theta_{i}<\left(\theta_{i, \min }+\delta_{i}\right), \\
0 & \text { otherwise. }\end{cases}
\end{aligned}
$$

One simple way how to use $V_{l}(\boldsymbol{\theta})$ for joint limit avoidance is to superimpose it with (12). In case that the joint limit avoidance should effect only the redundant nullspace motion, then instead of (2) one should use a controller of the form

$$
\boldsymbol{\tau}=\boldsymbol{g}(\boldsymbol{\theta})-\left(\frac{\partial V(\boldsymbol{\theta})}{\partial \boldsymbol{\theta}}\right)^{T}-\mathcal{N}(\boldsymbol{\theta})\left(\frac{\partial V_{l}(\boldsymbol{\theta})}{\partial \boldsymbol{\theta}}\right)^{T}-\boldsymbol{D}(\boldsymbol{\theta}) \dot{\boldsymbol{\theta}},
$$

where $\mathcal{N}(\boldsymbol{\theta})$ is a nullspace projection matrix which projects the differential of $V_{l}(\boldsymbol{\theta})$ onto the complement of the range space of the transposed body Jacobian matrices associated with the virtual object poses $\boldsymbol{H}_{o, r}(\boldsymbol{\theta})$ and $\boldsymbol{H}_{o, l}(\boldsymbol{\theta})^{6}$. Such kind of nullspace projections are in fact widely used in the literature on the control of redundant robots. But it should be mentioned that in contrast to (2) the control law (14) generally will not be a passive mapping from $\dot{\theta}$ to $-\tau$. The passivity properties of (14) instead depend on the particular form of $\mathcal{N}(\boldsymbol{\theta})$.

\footnotetext{
${ }^{6}$ In the case of the impedance behavior of Section II-C only one of the poses is used for the nullspace projection.
}

\section{EXPERIMENTS}

The proposed impedance behaviors have been implemented and examined with DLR's humanoid manipulator Justin ( [8], Fig. 1). This system consists of two fourfingered 12DOF DLR hands [19] and two 7DOF lightweight arms LWR-III [20] mounted on a 3DOF movable torso. The hands and arms are mounted in a humanoid configuration with a right-handed and a left-handed handarm-system. All the 41 joints $^{7}$ of the torso-arm-hand-system have joint torque sensors in addition to the common motor position sensors. In the following experiments lower-level joint torque controllers ${ }^{8}$ are used which get as their set-points the torque resulting from (14). Since Justin has 41 torquecontrolled DOF it is cumbersome to evaluate all these torques for the experiments. Instead the generalized task space forces of selected springs are analyzed. For the potential function $V_{s}\left(\boldsymbol{H}_{1}(\boldsymbol{\theta}), \boldsymbol{H}_{2}(\boldsymbol{\theta}), \mathcal{K}_{i}\right)$ of a single spatial spring, $\boldsymbol{f}_{i, j} \in \mathbb{R}^{3}$ and $\boldsymbol{m}_{i, j} \in \mathbb{R}^{3}$ denote the Cartesian forces and torques at the frame $\boldsymbol{H}_{j}(\boldsymbol{\theta})$ and represented in frame $\boldsymbol{H}_{j}(\boldsymbol{\theta})$. In Appendix $\mathrm{B}$ it is shown in detail how these generalized forces are computed. Additionally, the four fingertip forces $f_{h c, i}$ of the connecting springs (9) $f_{h c, i}=K_{h c, i}\left(\left\|\Delta \boldsymbol{x}_{i}\right\|-l_{i, d}\right)$ [17] will be analyzed.

Two manipulation scenarios have been implemented: A) Unscrewing of a can and B) Fine manipulation of a box. In the first experiment the impedance behavior presented in Section II-C is evaluated in order to examine the coordination of the right arm and hand motion. In the second experiment a coordinated two-handed manipulation will be shown by applying the impedance behavior based on $V_{O}(\boldsymbol{\theta})$ to the complete humanoid manipulator system. In both experiments the parameters of the joint limit avoidance are set to $\delta_{i}=20^{\circ}$ and $k_{i}=2 \mathrm{Nm} / \mathrm{rad}$ for the joints of the hands $k_{i}=0$ for the arm and torso joints. All experiments also appear in the video appended to this paper.

\section{A. Hand-Arm Coordination: Unscrewing of a Can}

Figure 1 depicts Justin in the start position to open a can. The left hand holds the can with a joint-level impedance controlled power grasp and the left arm is position-controlled. The right hand is initially above the can. In this particular application only the right hand-arm-system is object-level impedance controlled. While the object forces have also been analyzed in [17] for the hand alone, herein the focus is put on the coordination of the arm and hand motion.

A sequence of object-level commands ${ }^{9}$ for opening the can is sent to the robot:

1) Approach can.

2) GRASP: Shorten rest length of connecting stiffness.

\footnotetext{
${ }^{7}$ Two additional joints of the pan-tilt unit for the camera head are not used in these experiments.

${ }^{8}$ Notice that the incorporation of the joint torque controllers in the analysis would allow to consider also the flexibility of the joints as it was done in [21], [22]. However, the treatment of this paper instead focuses on the rigidbody behavior and, therefore, the joint flexibility is neglected.

${ }^{9}$ Commands are variations of the virtual equilibrium frames or rest lengths. These commands are linearly interpolated.
} 
3) TURN: Rotation along the screw direction and jump to 6) if the lid was rotated two times.

4) RELEASE: Increase rest length of connecting stiffness to release object.

5) RETURN: Rotate against screwing direction while fingers are not in contact with the lid.

6) Remove lid by a lateral motion.

In order to demonstrate the performance of the selected control law a step in the rotation of $0.3 \mathrm{rad}$ was commanded instead of interpolating the commands. In Fig. 6 the right hand object impedance torque $\boldsymbol{m}_{h o, r}$ shows that the control law converges well damped to values smaller than $0.3 \mathrm{Nm}$. The right hand object impedance force $\boldsymbol{f}_{h o, r}$ varies less than $1 \mathrm{~N}$ during the pure object rotation indicating very small coupling of rotational and translational motion (see Fig. 7). Theoretically, $\boldsymbol{m}_{h o, r}$ and $\boldsymbol{f}_{h o, r}$ should converge to zero. However, due to uncompensated friction a residual error remains. The connecting forces of the right hand vary less than $0.5 \mathrm{~N}$ as shown in Fig. 8. Note that in this hand configuration two fingers are opposing each other explaining that their corresponding connecting forces have similar values.

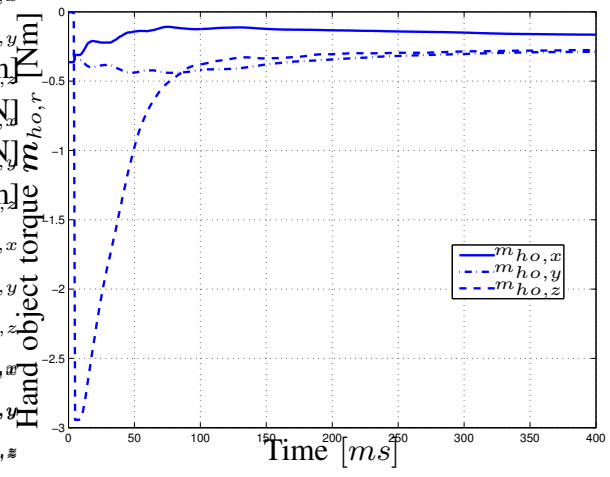

Fig. 6. Object impedance torque $\boldsymbol{m}_{h o, r}$ during the rotation around the object $z$-axis about $0.3 \mathrm{rad}$ using a rotational stiffness of $10 \mathrm{Nm} / \mathrm{rad}$.

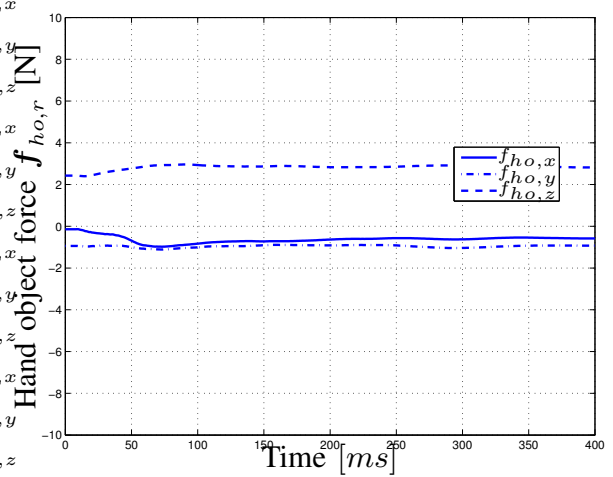

Fig. 7. Object impedance force $\boldsymbol{f}_{h o, r}$ during the rotation around the object $z$-axis about $0.3 \mathrm{rad}$ using a translational stiffness of $500 \mathrm{~N} / \mathrm{m}$.

Figure 9 illustrates the contribution of the arm and the hand towards the object motion. For simplicity we look at the Euclidean norm of the joint velocities at each time

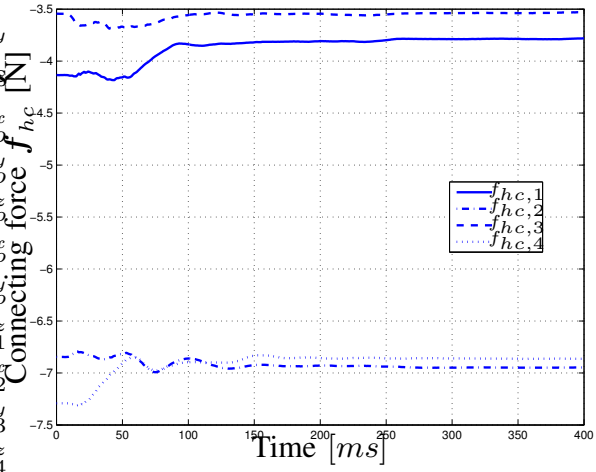

Fig. 8. Connecting forces $\boldsymbol{f}_{h c, r}$ during the rotation around the object $z$-axis about $0.3 \mathrm{rad}$ using $K_{h c, i}=300 \mathrm{~N} / \mathrm{m}$.

step $^{10}$. The hand joint velocities $\left\|\dot{\boldsymbol{\theta}}_{h}\right\|$ are bigger than the arm velocities $\left\|\dot{\boldsymbol{\theta}}_{a}\right\|$ by a factor of 40 . There are some major reasons:

- The static friction limit of the arm is higher compared to the hand's one.

- The response time of the arm joints is lower due to higher inertia and limited torque.

- The length of the robot links are roughly speaking hand : arm $=1: 10$.

- The configuration of the hand is suited very well for this step response and the nullspace component of equation (14) is small.

Furthermore, one can see that the fast part of the hand motion is finished much earlier than the arm motion.

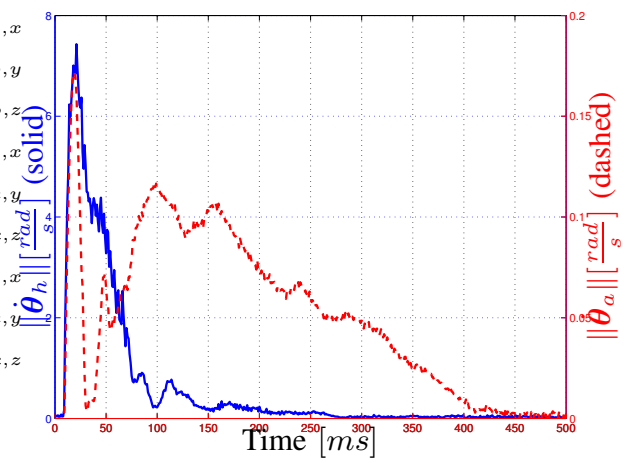

Fig. 9. Euclidean norm of the joint velocities of the arm $\left\|\dot{\boldsymbol{\theta}}_{a}\right\|$ (dashed) and the hand $\left\|\dot{\boldsymbol{\theta}}_{h}\right\|$ (solid) during the rotation around the object $z$-axis about $0.3 \mathrm{rad}$.

\section{B. Two-handed Coordination: Fine Manipulation of a Box}

In this experiment Justin is holding an object with contacts only at the fingertips of both hands (Fig. 10). We commanded translational motion on object-level using the control law (14) and the potential function (12) applied to the complete upper body system. Accordingly, all joints of Justin contributed to the object motion. The box is moved along different axes (see appended video). Again, we investigate

\footnotetext{
${ }^{10}$ The Euclidean norm gives a meaningful value for this comparison since all joints of Justin are revolute.
} 


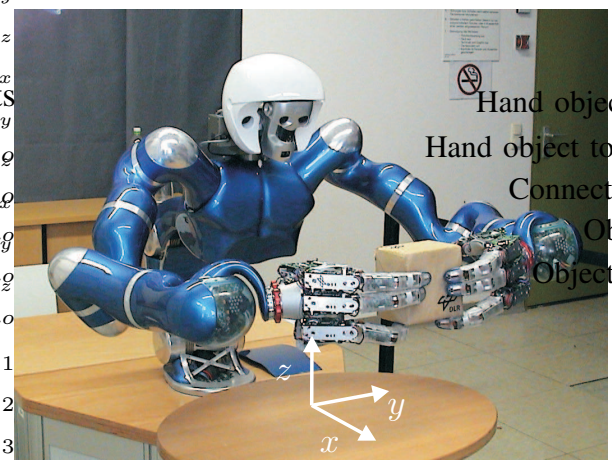

Fig. 10. Justin holding a box with two hands. The box is contacted only via fingertips.

the step response of the controller: A translation of $4 \mathrm{~cm}$ along the $z$-axis is commanded. Here, the object forces $\boldsymbol{f}_{o}$ and torques $\boldsymbol{m}_{o}$ are shown, which are the forces and torques from the spatial spring $\mathcal{K}_{o}$ rotated into the base frame. In Fig. 11 the object impedance force $f_{o}$ is reduced at a high rate which is mostly due to hand motion. The response contains an overshoot of about $5 \mathrm{~N}$ and converges after $500 \mathrm{~ms}$. Fig. 12 depicts low couplings with the rotational part $\boldsymbol{m}_{o}$ of the object stiffness that is perturbed less than $0.5 \mathrm{Nm}$. The coupling impedance forces $\boldsymbol{f}_{c, r}$ and $\boldsymbol{m}_{c, r}$ varies less than $4 \mathrm{~N}$ and $0.3 \mathrm{Nm}$, respectively, and converges to a steadystate (Fig. 13).

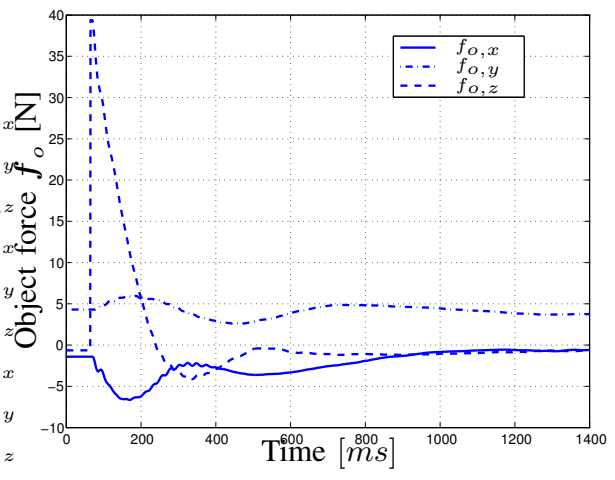

Fig. 11. Object impedance force $\boldsymbol{f}_{o}$ during box motion of $4 \mathrm{~cm}$ using a translational stiffness of $1000 \mathrm{~N} / \mathrm{m}$.

\section{Conclusions}

A two-arm control law consisting of a set of impedances was presented. First, separate Cartesian impedance controllers for the arms are combined with a compatible coupling impedance. Then an object-level control for the arms was discussed. An object-level control for a hand-arm system was presented and its combination with the two-arm controller led to the construction of a control law for twohanded manipulation. With this controller we can define the impedance of the grasped object and can command motions in 6D. Besides, only internal forces have to be parameterized by setting the coupling and connecting impedances. Two experiments, unscrewing a can and manipulation of a box,

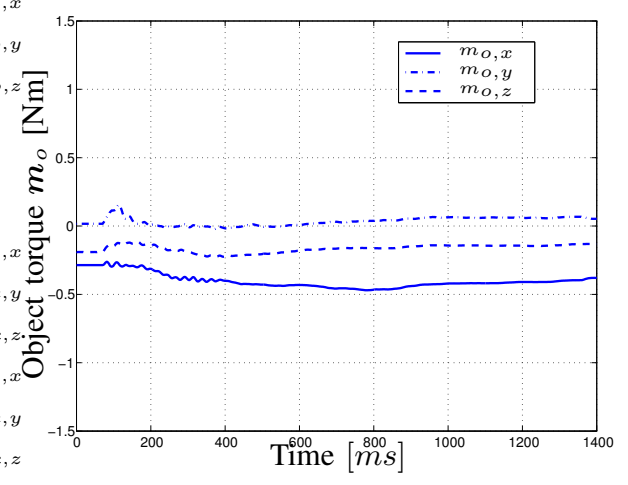

Fig. 12. Object impedance torque $\boldsymbol{m}_{o}$ during box motion of $4 \mathrm{~cm}$ using a rotational stiffness of $10 \mathrm{Nm} / \mathrm{rad}$.
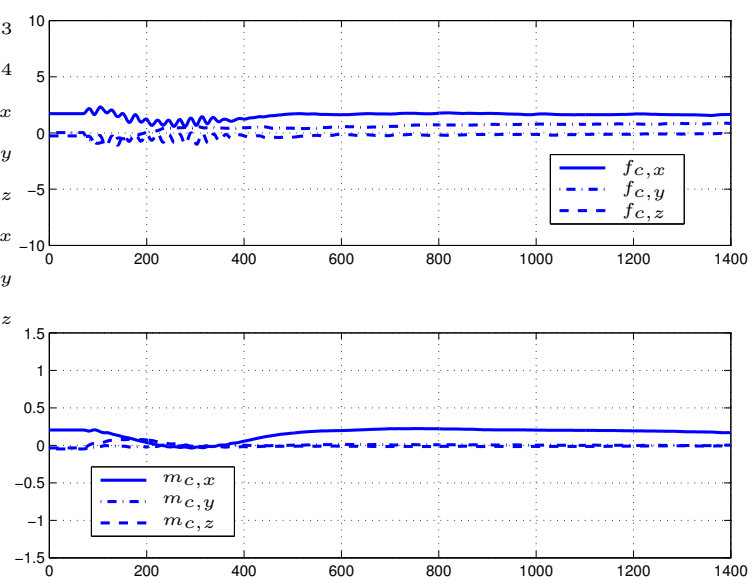

Fig. 13. Spatial coupling impedance force $\boldsymbol{f}_{c, r}$ and torque $\boldsymbol{m}_{c, r}$ during box motion of $4 \mathrm{~cm}$. The translational and rotational stiffness were chosen as $500 \mathrm{~N} / \mathrm{m}$ and $3 \mathrm{Nm} / \mathrm{rad}$, respectively.

prove the effectiveness of our approach as also can be seen in the appended video.

\section{APPENDIX}

\section{A - Example Implementation of a Spatial Spring}

In this appendix we provide one example implementation of a potential function related to a spatial spring between the frames $\boldsymbol{H}_{1}=\left[\boldsymbol{R}_{1}, \boldsymbol{p}_{1}\right]$ and $\boldsymbol{H}_{2}=\left[\boldsymbol{R}_{2}, \boldsymbol{p}_{2}\right]$.

The potential function is split up into a translational and a rotational part

$$
V_{s}\left(\boldsymbol{H}_{1}, \boldsymbol{H}_{2}, \mathcal{K}\right)=V_{t}\left(\boldsymbol{p}_{1}, \boldsymbol{p}_{2}, \mathcal{K}\right)+V_{r}\left(\boldsymbol{R}_{1}, \boldsymbol{R}_{2}, \mathcal{K}\right) .
$$

The translational part is simply chosen as

$$
V_{t}\left(\boldsymbol{p}_{1}, \boldsymbol{p}_{2}, \mathcal{K}\right)=\frac{1}{2} \boldsymbol{e}^{T} \boldsymbol{K}_{t} \boldsymbol{e},
$$

where $\boldsymbol{e}=\boldsymbol{p}_{1}-\boldsymbol{p}_{2}-\boldsymbol{p}_{12,0}$ is the translational deviation of the displacement $\boldsymbol{p}_{1}-\boldsymbol{p}_{2}$ from its rest length $\boldsymbol{p}_{12,0}$. The symmetric and positive definite matrix $\boldsymbol{K}_{t} \in \mathbb{R}^{3 \times 3}$ is the translational stiffness matrix. The rotational part is chosen as

$$
V_{r}\left(\boldsymbol{R}_{1}, \boldsymbol{R}_{2}, \mathcal{K}\right)=2 \boldsymbol{\epsilon}^{T} \boldsymbol{K}_{r} \boldsymbol{\epsilon}
$$


where $\epsilon$ is the vector part of the unit quaternion representation [10] of $\boldsymbol{R}_{1 v, 2 v}=\boldsymbol{R}_{1,1 v}^{T} \boldsymbol{R}_{1}^{T} \boldsymbol{R}_{2} \boldsymbol{R}_{2,2 v}$ (see Fig. 14). The rotation matrices $\boldsymbol{R}_{1,1 v}, \boldsymbol{R}_{2,2 v} \in S O(3)$ define the "rotational rest length" of the spring. The matrix $\boldsymbol{K}_{r} \in \mathbb{R}^{3 \times 3}$ represents the rotational stiffness matrix which is symmetric and positive definite.

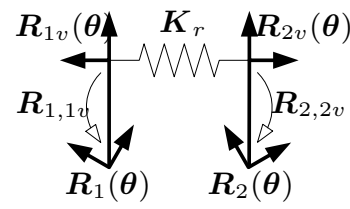

Fig. 14. Rotation matrices for constructing the rotational part of the spring.

The parameters $\mathcal{K}$ of this spatial spring are: The translational and rotational stiffness matrices $\boldsymbol{K}_{t}, \boldsymbol{K}_{r}$ as well as the translational and rotational rest length $\boldsymbol{p}_{12,0}, \boldsymbol{R}_{1,1 v}, \boldsymbol{R}_{2,2 v}$.

\section{B - Computation of the Cartesian Forces}

The Cartesian forces and torques are related to the differential of the potential function $\left.\partial V_{s}\left(\boldsymbol{H}_{1}(\boldsymbol{\theta}), \boldsymbol{H}_{2}(\boldsymbol{\theta}), \mathcal{K}_{i}\right)\right) / \partial \boldsymbol{\theta}:=\boldsymbol{\tau}_{i}(\boldsymbol{\theta})^{T}$ as follows. Clearly, one can write the differential in the form

$$
\begin{aligned}
\boldsymbol{\tau}_{i}(\boldsymbol{\theta})^{T}= & \underbrace{\sum_{j=1}^{2} \overbrace{\frac{\partial V_{s}\left(\boldsymbol{H}_{1}(\boldsymbol{\theta}), \boldsymbol{H}_{2}(\boldsymbol{\theta}), \mathcal{K}_{i}\right)}{\partial \boldsymbol{p}_{j}(\boldsymbol{\theta})}}^{\boldsymbol{f}_{i, j}^{\prime T}}}_{\boldsymbol{n}_{i}^{T}} \frac{\partial \boldsymbol{p}_{j}(\boldsymbol{\theta})}{\partial \boldsymbol{\theta}}+ \\
& \underbrace{\frac{\partial \boldsymbol{\epsilon}(\boldsymbol{\theta})}{\partial \boldsymbol{\theta}} .}_{\frac{\partial V_{s}\left(\boldsymbol{H}_{1}(\boldsymbol{\theta}), \boldsymbol{H}_{2}(\boldsymbol{\theta}), \mathcal{K}_{i}\right)}{\partial \boldsymbol{\epsilon}(\boldsymbol{\theta})}}
\end{aligned}
$$

The time derivative of the vector part of the unit quaternions can be written as $\dot{\boldsymbol{\epsilon}}=\boldsymbol{J}_{\boldsymbol{\omega} \boldsymbol{\epsilon}}(\boldsymbol{\theta}) \boldsymbol{\omega}_{12}$, where $\boldsymbol{\omega}_{12}=\boldsymbol{\omega}_{2}-\boldsymbol{\omega}_{1}$ represents the relative angular velocity between the frames $\boldsymbol{H}_{1}(\boldsymbol{\theta})$ and $\boldsymbol{H}_{2}(\boldsymbol{\theta})$ and $\boldsymbol{J}_{\boldsymbol{\omega} \boldsymbol{\epsilon}}(\boldsymbol{\theta})$ is the Jacobian between the orientation representations. With this mapping and the principle of virtual work it is easy to see that the task space torques can be written as

$$
\boldsymbol{m}_{i, 1}^{\prime}=-\boldsymbol{J}_{\boldsymbol{\omega} \boldsymbol{\epsilon}}(\boldsymbol{\theta})^{T} \boldsymbol{n}_{i}, \boldsymbol{m}_{i, 2}^{\prime}=\boldsymbol{J}_{\boldsymbol{\omega} \boldsymbol{\epsilon}}(\boldsymbol{\theta})^{T} \boldsymbol{n}_{i} .
$$

The generalized task space forces analyzed in the experiments are computed by rotation of $\boldsymbol{f}_{i, j}^{\prime}$ and $\boldsymbol{m}_{i, j}^{\prime}$ in to the frame $\boldsymbol{H}_{j}(\boldsymbol{\theta})$ via

$$
\boldsymbol{m}_{i, j}=\boldsymbol{R}_{j}(\boldsymbol{\theta})^{T} \boldsymbol{m}_{i, j}^{\prime}, \boldsymbol{f}_{i, j}=\boldsymbol{R}_{j}(\boldsymbol{\theta})^{T} \boldsymbol{f}_{i, j}^{\prime} .
$$

\section{REFERENCES}

[1] J.-H. Bae, S. Arimoto, R. Ozawa, M. Sekimoto, and M. Yoshida, "A unified control scheme for a whole robotic arm-fingers system in grasping and manipulation," in IEEE International Conference on Robotics and Automation, 2006, pp. 2131-2136.

[2] K. Nagai and T. Yoshikawa, "Grasping and manipulation by arm/multifingered-hand mechanisms," in IEEE International Conference on Robotics and Automation, 1995, pp. 1040-1047.

[3] C. Melchiorri and J. Salisbury, "Exploiting the redundancy of a handarm robotic system," MIT, A.I. Memo 1261, 1990.
[4] M. Diftler, R. Ambrose, S. Goza, K. Tyree, and E. Huber, "Robonaut mobile autonomy: Initial experiments," in IEEE International Conference on Robotics and Automation, 2005, pp. 1437-1442.

[5] K. Yokoi, K. Nakashima, M. Kobayashi, H. Mihune, H. Hasunuma, Y. Yanagihara, T. Ueno, T. Gokyuu, and K. Endou, "A tele-operated humanoid operator," The International Journal of Robotics Research, vol. 25, no. 5-6, pp. 593-602, 2006.

[6] R. P. Jr., A. H. Fagg, and R. A. Grupen, "Manipulation gaits: Sequences of grasp control tasks," in IEEE International Conference on Robotics and Automation, 2004, pp. 801-806.

[7] H. Iwata, H. Hoshino, T. Morita, and S. Sugano, "Human-humanoid physical interaction realizing force following and task fulfillment," in IEEE/RSJ International Conference on Intelligent Robots and Systems, 2000.

[8] Ch. Ott, O. Eiberger, W. Friedl, B. Bäuml, U. Hillenbrand, Ch. Borst, A. Albu-Schäffer, B. Brunner, H. Hirschmüller, S. Kielhöfer, R. Konietschke, M. Suppa, T. Wimböck, F. Zacharias, and G. Hirzinger, "A humanoid two-arm system for dexterous manipulation," in IEEE-RAS International Conference on Humanoid Robots, Genova/Italy, 2006, pp. 276-283.

[9] F. Caccavale, C. Natale, B. Siciliano, and L. Villani, "Six-dof impedance control based on angle/axis representations," IEEE Transactions on Robotics and Automation, vol. 15, no. 2, pp. 289-299, 1999.

[10] C. Natale, Interaction Control of Robot Manipulators: Six-Degreesof-Freedom Tasks, ser. Springer Tracts in Advanced Robotics (STAR). Springer-Verlag, 2003, vol. 3.

[11] E. Fasse and J. Broenink, "A spatial impedance controller for robotic manipulation," IEEE Transactions on Robotics and Automation, vol. 13, no. 4, pp. 546-556, 1997.

[12] E. Fasse, "On the spatial compliance of robotic manipulators," ASME Journal of Dynamic Systems, Measurement, and Control, vol. 119, pp. 839-844, 1997.

[13] S. Zhang and E. D. Fasse, "Spatial compliance modeling using a quaternion-based potential function method," Multibody System Dynamics, vol. 4, pp. 75-101, 2000.

[14] S. Stramigioli and V. Duindam, "Variable spatial springs for robot control applications," in IEEE/RSJ International Conference on Intelligent Robots and Systems, 2001, pp. 1906-1911.

[15] S. Stramigioli, Modeling and IPC Control of Interactive Mechanical Systems: A Coordinate-free Approach, ser. Lecture Notes in Control and Information Sciences. Springer-Verlag, 2001, vol. 266.

[16] D. Harville, Matrix Algebra from a Statistician's Perspective. Springer-Verlag, 1997.

[17] T. Wimböck, Ch. Ott, and G. Hirzinger, "Passivity-based objectlevel impedance control for a multifingered hand," in IEEE/RSJ International Conference on Intelligent Robots and Systems, 2006, pp. $4621-4627$.

[18] S. A. Schneider and J. R. H. Cannon, "Object impedance control for cooperative manipulation: Theory and experimental results," IEEE Transactions on Robotics and Automation, vol. 8, no. 3, pp. 383-394, 1992.

[19] J. Butterfaß, M. Grebenstein, H. Liu, and G. Hirzinger, "DLR-Hand II: Next generation of a dextrous robot hand," in IEEE International Conference on Robotics and Automation, 2001.

[20] G. Hirzinger, N. Sporer, A. Albu-Schäffer, M. Hähnle, R. Krenn, A. Pascucci, and M. Schedl, "DLR's torque-controlled light weight robot III - are we reaching the technological limits now?" in IEEE International Conference on Robotics and Automation, 2002, pp. 1710-1716.

[21] Ch. Ott, A. Albu-Schäffer, A. Kugi, S. Stramigioli, and G. Hirzinger, "A passivity based cartesian impedance controller for flexible joint robots - part I: Torque feedback and gravity compensation," in IEEE International Conference on Robotics and Automation, 2004, pp. 2659-2665.

[22] A. Albu-Schäffer, Ch. Ott, and G. Hirzinger, "A passivity based cartesian impedance controller for flexible joint robots - part II: Full state feedback, impedance design and experiments," in IEEE International Conference on Robotics and Automation, 2004, pp. 2666-2672. 